\title{
Validación de un cuestionario para el estudio del comportamiento sexual, social y corporal, de adolescentes escolares
}

\author{
Alfredo Hidalgo-San Martín, MSP, (1-2) Ramiro Caballero-Hoyos, Dr en C $S_{1}^{(1)}$
} Alfredo Celis-de la Rosa, Dr en C, ${ }^{(1)}$ Bettylu Rasmussen-Cruz, MSP.(1)

\section{Hidalgo-San Martín A, Caballero-Hoyos R, Celis-de la Rosa A, Rasmussen-Cruz B. Validación de un cuestionario para el estudio del comportamiento sexual, social y corporal, de adolescentes escolares. Salud Publica Mex 2003;45 supl 1:S58-S72.}

\begin{abstract}
Resumen
Objetivo. Validar un cuestionario autoadministrado de comportamientos sexuales, sociales y corporales para adolescentes por nivel escolar y género. Material y métodos Instrumento de 26 preguntas construido según DeVellis, aplicado en un estudio longitudinal prospectivo, hecho en 1994 a 1243 alumnos y alumnas de secundaria y preparatoria en Guadalajara, Jalisco, México. Validez de constructo establecida por análisis factorial exploratorio y la confiabilidad por la prueba Alfa de Cronbach. Resultados. Aparecen cinco factores en hombres usando valores propios (eigenvalue) $>1$ y cinco en mujeres, forzando la mejor solución. Veinte factores explican entre 59.3 y $70.6 \%$ de varianza, con promedio de pesos factoriales de $0.63-0.75$, y con 13 factores diferentes. El promedio de Alfa de Cronbach fue de 0.87-0.93 y en 16 factores hubo valores de Alpha de 0.710 más. Conclusiones. Cuestionario para poblaciones equivalentes con validez de constructo y confiabilidad interna aceptables.
\end{abstract}

Palabras clave: comportamiento sexual adolescente; validación de cuestionario; confiabilidad interna; validez de constructo; adolescentes escolares; M éxico

\author{
Hidalgo-San Martín A, Caballero-Hoyos R, \\ Celis-de la Rosa A, Rasmussen-Cruz B. \\ Validity and reliability of an instrument \\ for assessing the social, \\ and corporal sexual behaviors among school adolescents. \\ Salud Publica Mex 2003;45 suppl 1:S58-S72.
}

\begin{abstract}
A bstract
Objective. To validate a self-administered questionnaire about social and corporal sexual behaviors among adolescents, by school level and gender. Material and Methods The data collection instrument was constructed according to DeVellis and consisted of 26 items. It was used in a prospective longitudinal study conducted in 1994 in G uadalajara, Mexico, among 1243 secondary and high school students. The construct validity of the instrument was established using exploratory factorial analysis, and its reliability using the Cronbach's alpha test. Results. Five factors were obtained in males and an equal number in women, using eigenvalues $>1$ and by forcing the best solution. The explained variance was 59.3-70.6\%; the factorial weight average was $0.63-0.75$, and 13 different factors were selected. The Cronbach's alpha mean was 0.87-0.93 and 16 factors had Cronbach's alpha over 0.70 . Conclusions. The instrumen has an acceptable validity and reliability for studying the sexual, social, and corporal behaviors in adolescents similar populations.
\end{abstract}

Key words: adolescent sexual behavior; questionnaire validation; reliability; construct validity; adolescents students; Mexico

(1) Unidad de Investigación Epidemiológica y en Servicios de Salud del Adolescente. Instituto Mexicano del Seguro Social. Guadalajara, Jalisco, México.

(2) Centro Universitario de Ciencias de la Salud de la Universidad de Guadalajara. Guadalajara, Jalisco, México.

Fecha de recibido: 4 de julio de 2000 - Fecha de aprobado: 24 de enero de 2001

Solicitud de sobretiros: Dr. Alfredo Hidalgo-San Martín, A partado 31-178 Guadalajara, Jalisco 45060. Guadalajara, Jalisco, México. Correo electrónico: ahidalgo@ udgserv.cencar.udg.mx 
L a Organización Mundial de la Salud (OMS) señala que en el mundo contemporáneo las condiciones de vida para mucha gente joven han cambiado y con ello su patrón de conducta sexual, manifestado en una pubertad más temprana, matrimonio más tardío, menor control y más autonomía de su familia y una intensa exposición al erotismo de los medios de comunicación. ${ }^{1,2}$ Estos cambios han aumentado el riesgo de embarazo y enfermedades de transmisión sexual. ${ }^{2}$ Para prevenir problemas como los señalados y promover una educación acorde al proceso de cambio en adolescentes, se hace indispensable el estudio del desarrollo de sus comportamientos sexuales.

El proceso de desarrollo de comportamientos sexuales puede ser abordado desde la teoría de la Secuencia del Comportamiento Sexual de Byrne, que intenta integrar los enfoques biológico, social y psicosexual en unidades de secuencia de comportamientos sexuales que incluyen "una serie de procesos individuales de excitación, de afectividad y cognitivos que son afectados por fuerzas culturales o de la estructura social y a su vez son causa de los comportamientos sexuales individuales." ${ }^{3}$ Fisher aborda este modelo en la adolescencia, señalando que desde el periodo de pubertad aparecen cambios que implican dominios individuales o fisiológicos ("excitación sexual que se integra con procesos afectivos y cognitivos haciendo prominente las fantasías") y sociales ("los sentimientos, evaluaciones, creencias, expectativas y fantasías son conformadas por las interacciones sociales que se van produciendo durante la adolescencia"). ${ }^{4}$ De esa manera, bajo el enfoque anterior, podríamos considerar comportamientos sexuales relacionados con el dominio de excitación o "corporal" y otros con el dominio de interacción o "social".

Sorensen, en Estados Unidos de América (EUA) inició en los años setenta la investigación en los adolescentes en cuanto a sus relaciones coitales premaritales, monogámicas y homosexuales, y sus prácticas anticonceptivas. ${ }^{5}$ Su estudio fue seguido de muchos semejantes, referidos especialmente a las relaciones coitales, pero sólo algunos pocos estudios focalizados en actividades previas al coito o en las dimensiones psicosociales. ${ }^{6}$

En cuanto al dominio "corporal", Collins en 1974, en universitarios australianos, escribió sobre los niveles de intimidad " (7); Rodgers en EUA con la encuesta longitudinal Adolescent Sexuality (ADSEX) en $1978^{8}$ señaló la "transición suave" de las conductas sexuales; Grace identificó secuencias, pero enfatizó que no todos las tenían iguales y un pequeño porcentaje no presentaba esas conductas, ${ }^{9}$ y Roche detalló diversas conductas..$^{10}$ Los comportamientos que aparecieron en estos estudios fueron "tomarse de manos", "besos o besuqueo", "abrazos", "beso y abrazo prolongado" "caricias sobre la ropa" (o "caricias ligeras") o "caricias suaves" o "tocar los pechos sobre la ropa ", "tocar los pechos bajo la ropa", "caricias bajo ropa" (o "caricias pesadas o intensas"), "sentir los órganos femeninos directamente", "sentir el pene directamente", sexo oral, "masturbación mutua" y relación coital , coito o cópula.

En el dominio social de interacción se han identificado comportamientos afines por Silva ${ }^{1}$ en Chile, estudiando la búsqueda de pareja, ${ }_{11}^{11}$ por el mismo Roche $^{10}$ y por Montgomery, quien revisó la literatura e investigó experiencias románticas de enamoramiento y señaló que pocos trabajos han estudiado específicamente la emergencia, desarrollo y consecuencias psicosociales de las relaciones románticas tempranas. ${ }^{12}$ Los comportamientos encontrados fueron "salir con una pareja sin afecto", "salir con una pareja con afecto", ",andar con" (predominio de la intimidad física), "salir enamorado con una pareja", "salir enamorado sólo con una persona" "pololeo" (con intimidad emocional que implica un compartir y explorar los sentimientos, pensamientos, valores y metas de la vida en pareja") y "compromiso", "noviazgo" y "matrimonio".

En México se abordaron preferentemente las relaciones sexuales y la anticoncepción en relación con conocimientos, actitudes y prácticas. La Encuesta Nacional sobre Sexualidad y Familia en Jóvenes de Educación Media Superior del Consejo Nacional de Población (Conapo), exploró en más de 10000 jóvenes de 14 a 25 años de edad sus actitudes sexuales, medios de información, conocimientos, actitudes ante las relaciones sexuales y uso de anticonceptivos. ${ }^{13}$ Morris y colaboradores estudiaron en dos delegaciones de México D.F., en 1987, a 3000 adolescentes y jóvenes entre 10 y 25 años de edad, enfocándose a las relaciones sexuales, pareja, uso de anticonceptivos y edad de inicio. ${ }^{14}$ La Encuesta sobre Comportamiento Reproductivo entre adolescentes y jóvenes del área metropolitana de la Ciudad de México en 1987 fue con igual enfoque hacia la presencia de relaciones sexuales y el uso de anticonceptivos, ${ }^{15}$ el estudio de CONASIDA (1992-1993) en la Ciudad de México se hizo con un énfasis en comportamientos en torno a las relaciones sexuales coitales y sus riesgos, ${ }^{16}$ y el estudio de Fleiz y colaboradores, en 1997, investigó las relaciones sexuales y el uso de métodos contraceptivos en una muestra de más de 10000 escolares de enseñanza media y media superior de la Ciudad de México. ${ }^{17}$

En relación con la validación de instrumentos para estudios de comportamientos sexuales, Daré en 1999 realizó una revisión de la confiabilidad de cuestiona- 
rios para comportamientos sexuales autorreportados desde los años 40 en diversas poblaciones, incluyendo adolescentes. Encontró que las respuestas estuvieron afectadas por factores sociales, culturales y regionales y que las conductas que son socialmente inaceptables fueron sobrerreportadas. ${ }^{18}$ Winhardt y colaboradores ${ }^{19}$ publicaron un estudio sobre los autorreportes de 1990 a 1998 relacionados con el riesgo de infección por VIH, señalando sesgos como el subreporte de conductas estigmatizadas y el sobrerreporte de conductas normadas. Consideró que se continúa usando esta técnica, dada las dificultades para contar con otros métodos más "duros". Señaló que Boekelo encontró que igual son de confiables la entrevista y el cuestionario autoaplicado. ${ }^{19}$

Puesto que no existe en México un instrumento validado y publicado que explore en adolescentes el desarrollo de los comportamientos sexuales hasta llegar al coito, tanto en las relaciones físicas como sociales, nuestro objetivo fue validar un cuestionario de autoadministración acerca de comportamientos sexuales, sociales y corporales, en adolescentes de secundaria y de preparatoria, en Guadalajara, México, y comparar las semejanzas y diferencias en los hallazgos entre niveles escolares y por géneros.

\section{Material y métodos}

Con el fin de aplicar un instrumento de estudio se escogieron, por la disposición positiva de sus autoridades para este tipo de investigaciones, una escuela secundaria y otra preparatoria, ambas públicas, ubicadas en una area residencial e industrial de Guadalajara, México. Para elaborar el instrumento se tomaron en consideración los siguientes pasos para construir escalas, propuestos por De Vellis: ${ }^{20}$

1. Definir qué se medirá, con apoyo en la teoría y con un nivel de especificidad operante

2. Generar un grupo de items, que reflejen el propósito de la escala, preferiblemente con redundancia, con características de buen item, y equilibrando las tendencias (positiva-negativa)

3. Establecer sus formatos de medición, mediante tipos de escala o items y el marco temporal del asunto

4. Revisar el conjunto de items por expertos, para confirmar la definición del fenómeno, evaluar la claridad y la concisión y sugerir alternativas mejores

5. Considerar la inclusión de items de validación, para detectar respuestas socialmente deseables o comparar con otra escala relacionada
6. Aplicarlos a una muestra poblacional grande, representativa y

7. Evaluar los items, en cuanto puedan constituir una escala por estar intercorrelacionados, con varianzas altas, promedios centrales, confiabilidad adecuada, e identificar los set de items que conforman diversas variables latentes.

En el primer paso se consideró que, con la teoría de la Secuencia del Comportamiento sexual de Byrne, ${ }^{3}$ y con el abordaje de componentes individuales y sociales que Fisher ${ }^{4}$ hace en adolescentes, el constructo comportamiento sexual pudo trabajarse con la definición de Katchadourian, ${ }^{21}$ quien lo describe como "aquella actividad claramente discernible en un individuo, incluyendo tanto los fenómenos observables como los informes verbales que se refieren a estados o experiencias subjetivas relacionadas a la sexualidad".

Para el segundo paso, se utilizó la teoría de referen$\mathrm{cia}^{3,4}$ la literatura empírica, ${ }^{7-16}$ y la experiencia de los autores. Además, la cultura adolescente ${ }^{18}$ se consideró mediante entrevistas a una pareja adolescente y a un pequeño grupo de adolescentes escolares, quienes describieron comportamientos sexuales de ellos y sus pares, revisaron la comprensibilidad y propusieron el uso de términos populares en los items. La agrupación de items resultantes llevó a establecer dos dominios de comportamientos sexuales, uno social y otro corporal y diversas dimensiones en su interior: a) el Dominio social incluyó las dimensiones: Fantasías, Imágenes o expresiones corporales o interacciones con la persona deseada, su Búsqueda, Noviazgo informal y Noviazgo formal y b) el Dominio corporal incluyó las dimensiones: Sensaciones autoeróticas, físicas, espontáneas o provocadas de exploración del propio cuerpo, Contactos externos físicos y Contactos íntimos con la pareja deseada. Se logró un total de 51 items.

Para asegurar el control de errores de medición ${ }^{19,22}$ se buscó construir items claros, uso de lenguaje popular, items neutros y se los ordenó de los más públicos a los más privados.

Paralelamente, para el tercer paso, del total de items, se estructuró un grupo de 26 en una escala de medición ordinal basada en el tiempo de ocurrencia de los comportamientos (anexo 1).

El cuarto paso consistió en su revisión por 15 expertos en adolescencia o sexualidad, locales y del resto del país, quienes identificaron si los items correspondían y si cubrían los dominios social o corporal y las dimensiones temáticas inicialmente formuladas. Se encontró un porcentaje de correspondencia de ubicación de items en los dominios y dimensiones aceptable: para los comportamientos sexuales sociales fue 
de $61.2 \%$ (con un rango de $40 \%$ a $86.6 \%$ ) y para comportamiento sexual corporal fue de $78 \%$ (con un rango de $46.6 \%$ a $100 \%$ ). No alcanzó a $1 \%$ la selección de otros o no clasificable. Los expertos confirmaron la adecuación de los items propuestos y con ello la definición del fenómeno en estudio.

En relación con el quinto paso se consideró analizar la probabilidad de subregistro o sobrerregistro en los items, dependiendo del género, dadas las normatividades sociales existentes: subregistro de masturbación en mujeres por considerarse anormal para ellas y sobrerregistro en el hombre, por ser hombre. Se analizó mediante la prueba de ji cuadrada, comparando en ambos géneros la asociación entre masturbación y mediante una pregunta de control ("La masturbación en un(a) adolescente indica: 1. Un desarrollo normal 2. Un desarrollo en el límite de lo normal 3. Un trastorno sexual y 4 . No sé").

Para el sexto paso se aplicó el cuestionario, primero en una prueba piloto, a 110 alumnos de uno u otro sexo de las mismas escuelas secundaria y preparatoria, pero de un grado superior a las cohortes a seleccionar, con el fin de explorar sobre la claridad y comprensión de las preguntas y la duración de la prueba. Se encontraron mayores dificultades de comprensión en secundaria, por lo que se integraron los términos populares sugeridos. Además, considerando las diferencias de género, se conformaron cuestionarios para cada uno.

Posteriormente, los cuestionarios finales (anexo 1), siguiendo un procedimiento estandarizado por los aplicadores a la población de estudio, se autoadministraron en 1994 a 1243 alumnos correspondientes a cuatro grupos de estudio: 209 mujeres y 274 hombres de primer año de secundaria, y 367 mujeres y 393 hombres de primer año de preparatoria. Estos grupos pertenecían a la población basal de una investigación longitudinal, motivo por el cual los alumnos fueron a partir del total de las cohortes 1994-1997.

Para asegurar el control de errores en la aplicación, ${ }^{18,22}$ se usaron encuestadores externos que estandarizaron, sin la presencia del maestro, en los mismos salones donde aplicaban sus pruebas, dentro del tiempo de una hora de clase, a mitad de la mañana y de la tarde, para controlar la fatiga. Se aplicó una clave secreta para el estudio longitudinal tipo panel, lo que permitió comparar a los que repetían la clave en las aplicaciones siguientes, con los que no, y estudiar si eso afectaba sus respuestas sobre comportamientos sexuales. Al iniciar la aplicación se hizo una introducción que hablaba de lo natural del comportamiento sexual en el desarrollo adolescente, del objetivo colectivo y no personal, de ser una participación voluntaria y anónima mediante una clave secreta, para emplearse en el estudio longitudinal de tipo panel y se separó a hombres de mujeres para impedir bloqueos.

Se incluyeron alumnos de primer ingreso, comprendidos entre los 10 y 19 años, de las escuelas seleccionadas; se excluyeron los alumnos de primer ingreso que no acudieron al salón, los que no desearon participar (aproximadamente 3\%) o llegaron atrasados (menos de $1 \%$ ). Se eliminaron los alumnos de 10 a 19 años que no llenaron adecuadamente el cuestionario (menos de 1\%).

Finalmente, el séptimo paso implicó realizar propiamente la validación del instrumento. Este, por referirse a un fenómeno psicosocial en una población específica que buscaba identificar las variables latentes para agrupar los comportamientos sexuales estudiados, exigía la validez de constructo, a través de un análisis factorial exploratorio y la confiabilidad por consistencia interna de los factores encontrados.

Para decidir el uso del análisis factorial se empleó el índice Kayser-Meyer-Olkin (KMO), que compara las magnitudes de los coeficientes de correlación observados entre las variables (que estima factores comunes), con las magnitudes de los coeficientes de correlación parcial entre pares de variables (que estima factores únicos). Si el valor es alto indica que la intercorrelación entre las variables es grande y el análisis factorial es adecuado (0.70 o más) , si es bajo no procede, porque habría tantos factores como variables. ${ }^{23} \mathrm{El}$ análisis factorial se utilizó con un objetivo exploratorio para identificar, a partir de los 26 items, en los cuestionarios por género de cada grupo de estudio, algunos pocos factores que explicaran el máximo de variabilidad y que se estructuraran con variables (items) específicas al factor. Las variables se tratarían con análisis factorial, ya que eran ordinales con valores numéricos que no distorsionaban la propiedad de tiempo de ocurrencia del comportamiento. ${ }^{24}$

Para la extracción de factores se usó el método de componentes principales que se emplea dentro del análisis factorial, porque usa algoritmos similares y porque una estadística generada con el análisis de componentes sirve como el medio más práctico de resolución de problemas de factorización, como por ejemplo usando el criterio de valor propio mayor de 1 para generar factores. ${ }^{24}$ Para obtener factores que integran variables estrechamente correlacionadas, se usó el procedimiento de rotación de factores varimax con un valor propio (varianza explicada) de 1 o más. Para lograr la ubicación de los items en un solo factor, con el fin de una descripción parsimoniosa, ${ }^{25}$ se buscó como punto de corte aquel valor de peso factorial que la produjera con el menor número de factores ${ }^{24} \mathrm{y}$ en los valores repetidos en otros factores fueran del menor 
valor, siguiendo a Nunally, ${ }^{26}$ quien establece que "cada factor debe tener algunas variables que se correlacionen con él casi exclusivamente (la mayoría de las variables que se correlacionan altamente con este factor no deben correlacionarse más de 0.3 con cualquier otro factor, y las variables marcadoras cuya inclusión se destaca deben correlacionarse por encima de 0.5). Los resultados obtenidos para las escalas del instrumento se expresaron en número de factores extraídos (por valores propios mayores a 1 o por pesos factoriales forzados) y pesos factoriales promedio que reflejaron el peso de las variables en la medición del factor. Los valores propios de los factores resultantes explicaron el porcentaje total acumulado de la varianza, pero por ser rotados, el valor propio de cada factor no nos indicó la importancia relativa de cada uno. ${ }^{24}$

La confiabilidad se estudió mediante la consistencia interna, basada en el grado de homogeneidad de las preguntas de un factor, expresada en el Coeficiente de Alfa de Cronbach, que indica la proporción de varianza en los puntajes de la escala que es atribuida al puntaje verdadero. ${ }^{27}$ Se estudió la correlación de items con los factores. Se buscó una alta varianza del item que demostrara la variabilidad entre los individuos para contestar, e idealmente que el promedio se acercara al centro del rango para alcanzar mayor varianza y tener mayor relación con otros. Los valores del coeficiente debían encontrarse entre 0.0 y 1.0. De acuerdo a Nunally son aceptables los valores de 0.70 o más, y en los de 0.90 o más, se debería considerar que hay redundancias que requiere acortar la escala. Se consideró la eliminación de items cuya presencia afectara en forma importante el valor de alpha. ${ }^{26}$

Para realizar ambos análisis se empleó el paquete SPSS, versión $6.0 .^{28}$

\section{Consideraciones éticas}

Se informó a los participantes de los objetivos y métodos de trabajo para que pudieran elegir participar o no, sin implicación alguna para sus estudios, y se informó a las autoridades de las escuelas, y por intermedio de ellas, los padres de alumnos de secundaria recibieron una comunicación en la cual se anexa una información de los investigadores para darles a conocer sobre la aplicación, sus objetivos y métodos. En el caso de no aceptar, los padres debían informarlo a la dirección para no incluirlo. Alumnos y alumnas de secundaria y preparatoria firmaron una carta de consentimiento informado después de leer el cuestionario. El anonimato se cubrió con una clave secreta que se utilizó para el estudio longitudinal tipo panel..$^{29}$

\section{Resultados}

\section{Datos sociodemográficos}

Los promedios de edad y sus desviaciones estándar fueron los siguientes: en mujeres de secundaria (Ms) 12.7 \pm 0.70 ; en hombres de secundaria (Hs) 12.8 \pm 0.75 ; en mujeres de preparatoria (Mp) 16.5 \pm 1.1 y en hombres de preparatoria (Hp) 16.3 \pm 1.09 . Los estudiantes que trabajaban eran Ms (7.7\%), Hs (28.1\%), Mp (16.39\%) y Hp (31.1\%). El porcentaje de estudiantes que vivían con ambos padres fue el siguiente: 87.1 para Ms, 87.6 para Hs, 83.4 para Mp y 87.1 para Hp. Los estudiantes cuyas madres cursaron preparatoria o estudios profesionales fueron Ms (15.3\%), Hs (16.9\%), Mp (18.5\% y Hp (18.2). Estudiantes con madres amas de casa: Ms (63.6\%), Hs (69.8\%), Mp (69.1\%) y Hp (70.1\%). Estudiantes cuyos padres desempeñaban algún trabajo manual: Ms (25.8\%), Hs (25.9\%) Mp (21.7\%) y Hp $23.5 \%)$. Estudiantes con padres trabajadores por cuenta propia o empleados: Ms (47.8\%), Hs (56.2\%), Mp (45.5\%) y Hp (47\%). Estudiantes con padres profesionales o directivos: Ms (6.2\%), Hs (9.0\%), Mp (4.0\%) y Hp 18.1\%).

\section{Validez de constructo}

Para aplicar análisis factorial se estudiaron los índices $\mathrm{KMO}$, que fueron superiores a 0.7 (en mujeres de secundaria, 0.92 y en las de preparatoria, 0.86 ; en hombres de secundaria 0.91 y en los de preparatoria 0.91 ). Se identificaron cinco factores en cada uno de los dos grupos de hombres a partir de valores propios iguales o mayor de 1 , con punto de corte de 0.50 y otros cinco factores en cada uno de los dos grupos de mujeres de secundaria y preparatoria, con puntos de corte de 0.45 y 0.46 de peso factorial, respectivamente (cuadro I). Los cinco factores en cada grupo explicaron entre $59.3 \%$ y $70.6 \%$ de la varianza total y con un promedio de pesos factoriales entre 0.63 y 0.75 . Del total de 20 factores, en los cuatro grupos, hubo 13 que fueron diferentes, equivaliendo cada uno a una variable latente específica en la población total. Les denominamos factores I al XIII.

Los factores se distribuyeron al interior de los dos dominios, el social y el corporal, excepto en mujeres de secundaria, en las cuales un factor (VII) integró dimensiones del dominio social (Noviazgo formal) y del dominio corporal (Contacto externo). Al analizarlos por dominios y dimensiones (con letra mayúscula inicial) y comportamientos (en cursiva) se encontró: a) un Dominio social constituido por las di- 


\section{Cuadro I \\ Perfil de factores identificados según dominios, DIMENSIONES Y COMPORTAMIENTOS POR GRUPOS de estudio. Guadalajara, Jalisco, México}

Dominios, dimensiones

y comportamientos

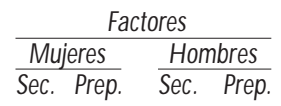

SO CIAL

Item Fantasía

1 Idea de estar solo

2 Escrito de lo que sientes

Imagen

3 Te vea arreglado(a)

4 Aparecer sexy

5 Hacer cosas por

Búsqueda

6 Conquistar

7 Llamar (teléfono o recados)

N oviazgo informal

8 Salir solos

9 Juntarse para acariciarse

$\mathrm{N}$ oviazgo formal

10 Noviazgo formal

CORPORAL

Contacto externo

Tomar de la mano

Acariciar partes externas

Besar suavemente

Excitación en baile

Sensaciones autoeróticas

Sensación física

Sueños excitantes

Masturbación

Contacto íntimo no genital

Caricias de senos encima de ropa

Caricias de senos debajo de ropa

$C$ aricias genitales encima de ropa

Caricias genitales debajo de ropa

Besar apasionado

Contacto íntimo genital

Caricias mutuas de genitales

Contacto genital sin introducir

Contacto genital con introducir sin eyaculación

26 Contacto genital con introducir con eyaculación (coito)

$N$ úmero de factores

Punto de corte de peso factorial

$\%$ Varianza total explicada

Promedio peso factorial mensiones del factor I o de Fantasía, Imagen y Búsqueda de Ms; del factor II; o de Fantasía e Imagen y Conquistar de Mp; del factor III; o de Fantasía e Imagen sin Hacer cosas por, de Hs y Hp; del factor IV o de Noviazgo informal de Ms; del factor $\mathrm{V}$ o de Noviazgo informal, Noviazgo formal y Llamar de Mp y del factor VI o de Noviazgo informal, Noviazgo formal, Búsqueda y Hacer cosas por Hs y Hp, y b), un Dominio corporal constituido por el factor VII o de Contacto externo, Noviazgo formal, Sensación física y Besar apasionado de Ms; el factor VIII o de Contacto externo de Hs y Mp; el factor IX o de Contacto externo sin Excitación en baile de Hp; el factor $\mathrm{X}$ o de Sensaciones autoeróticas sin Sensación física y con Excitación en baile DE Ms; el factor XI o de Sensaciones autoeróticas de Hs y Mp; el factor XII, que incluyó Sensaciones autoeróticas y Excitación en baile de $\mathrm{Hp}$ y el factor XIII o de Contacto íntimo no genital y genital de Ms, Mp, $\mathrm{Hs}$ y $\mathrm{Hp}$, que fueron las únicas dimensiones compartidas con iguales comportamientos por los cuatro grupos.

El perfil de factores en estos cuatro grupos (cuadro I) mostró que se estructura en forma diferente en las mujeres y casi semejante en los hombres, lo que generó cinco espacios factoriales o de contenido que se pueden describir como

a) Un primer espacio con tres dimensiones: Fantasía, Imagen y Búsqueda en Ms, que se redujeron hasta las dos primeras en los hombres

b) Un segundo espacio con la dimensión Noviazgo informal en Ms, que se amplió hasta integrarse con Búsqueda y Noviazgo formal en los hombres

c) Un tercer espacio con las dimensiones Contacto externo, Noviazgo formal y parcialmente Sensaciones autoeróticas en Ms, que se redujeron hasta Contacto externo en Mpa Hp

d) Un cuarto espacio de las Sensaciones autoeróticas parciales en Ms, que se completó en Mpa Hp y

e) El quinto espacio de Contacto íntimo, que se mantuvo sin variación en los cuatro grupos.

En cuanto a cada género, entre los hombres los factores fueron semejantes con una excepción (Sensaciones autoeróticas que en preparatoria incluyó la Excitación en baile). Por el contrario, entre las mujeres, donde la factorización fue diversa, en secundaria separó el Noviazgo informal del Noviazgo formal (en factores VII y IV); no así en la preparatoria, que los integró en el factor V). La factorización en secundaria integró en el Noviazgo formal el Contacto externo (en factor VII), mientras que en preparatoria los separó. 
Al comparar entre géneros se observó una factorización semejante (factores VIII, XI y XIII) entre mujeres de preparatoria y hombres de secundaria, $y$ diferencial entre las mujeres, -donde la dimensión de Fantasía e Imagen se asoció con la Búsqueda (factor I y
II)-, y en los hombres, en donde no hay esta asociación y la Búsqueda se integró con el Noviazgo informal y el formal (factor VI).

En los cuadros II a V se presentan, en cada grupo de estudio, para cada factor encontrado, los pesos

\section{Cuadro II \\ FACTORES IDENTIFICADOS SEGÚN DOMINIOS, DIMENSIONES Y COMPORTAMIENTOS EN MUJERES DE SECUNDARIA. Guadalajara, Jalisco, México}

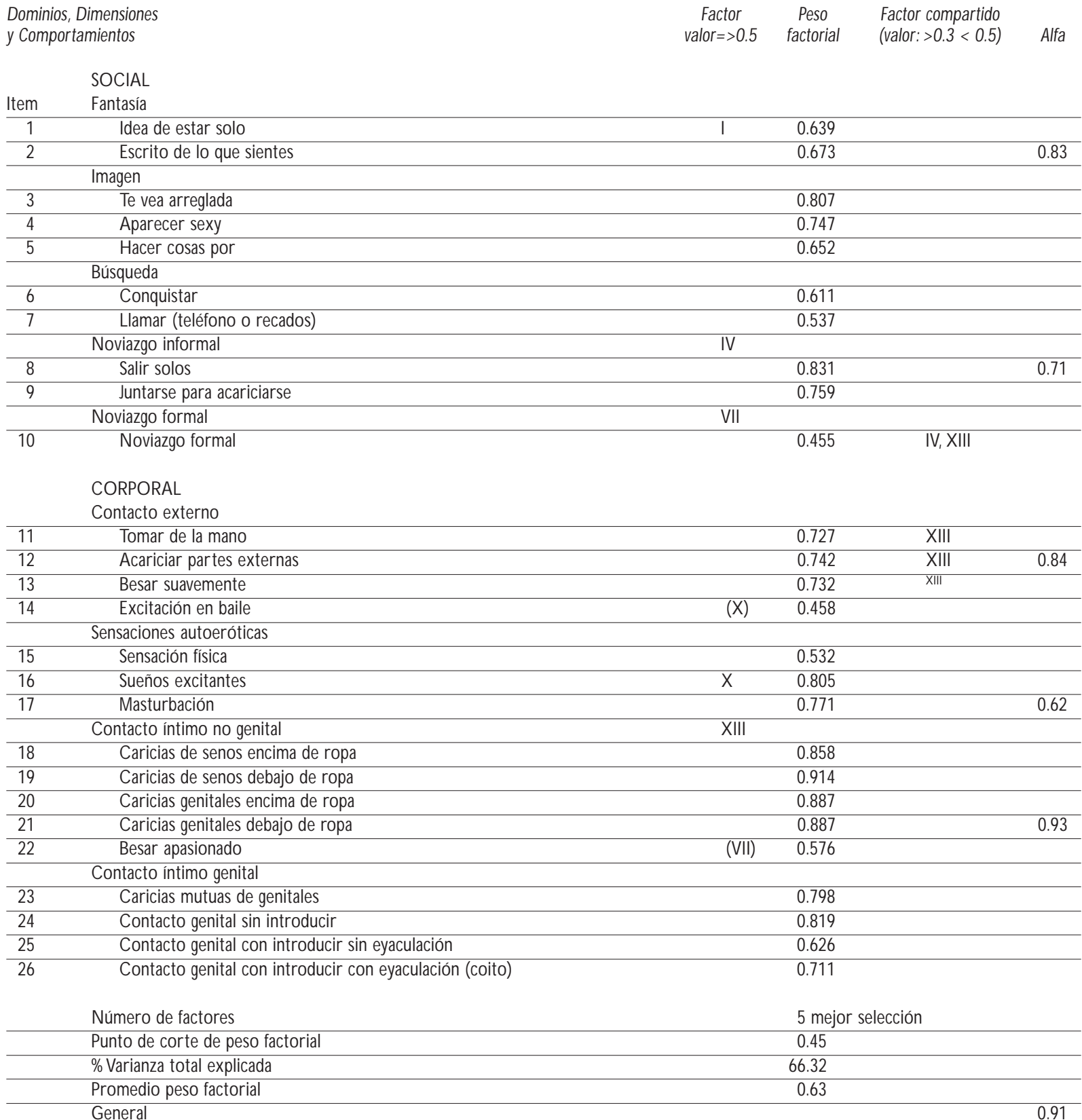

(N úmero romano $)$ factor correspondiente a un comportamiento que está compartido 
factoriales después de la rotación de los items que lo componen, el o los factores compartidos y el valor de Alfa de Cronbach. Cuando se analizan los 104 items que suman los cuatro grupos, encontramos que $25 \%$ se ubicaron no sólo en el factor donde alcanzaron el más alto peso factorial, sino en uno o dos factores con pesos menores de $0.5 \mathrm{y}$ mayores de 0.3 .

En mujeres de secundaria (cuadro II ) se encuentran los factores I, IV, VII, X y XIII. Es el único grupo donde el Besar apasionado no forma parte de Contacto

\section{Cuadro III \\ FACTORES IDENTIFICADOS SEGÚN DOMINIOS, DIMENSIONES Y COMPORTAMIENTOS EN MUJERES DE PREPARATORIA. Guadalajara, Jalisco, México}

Dominios, Dimensiones

y Comportamientos

SOCIAL

Item Fantasía

\begin{tabular}{|c|c|c|c|c|c|}
\hline 1 & Idea de estar solo & II & 0.564 & & \\
\hline 2 & Escrito de lo que sientes & & 0.630 & & 0.74 \\
\hline \multicolumn{6}{|c|}{ Imagen } \\
\hline 3 & Te vea arreglada & & 0.735 & & \\
\hline 4 & Aparecer sexy & & 0.664 & & \\
\hline 5 & Hacer cosas por & & 0.512 & $\mathrm{~V}, \mathrm{XI}$ & \\
\hline \multicolumn{6}{|c|}{ Búsqueda } \\
\hline 6 & Conquistar & & 0.496 & $\mathrm{~V}, \mathrm{XI}$ & \\
\hline 7 & Llamar (teléfono o recados) & V & 0.694 & II & \\
\hline \multicolumn{6}{|c|}{ N oviazgo informal } \\
\hline 8 & Salir solos & & 0.737 & & 0.65 \\
\hline 9 & Juntarse para acariciarse & & 0.598 & & \\
\hline \multicolumn{6}{|c|}{ Noviazgo formal } \\
\hline 10 & N oviazgo formal & & 0.658 & & \\
\hline
\end{tabular}

CORPORAL

Contacto externo

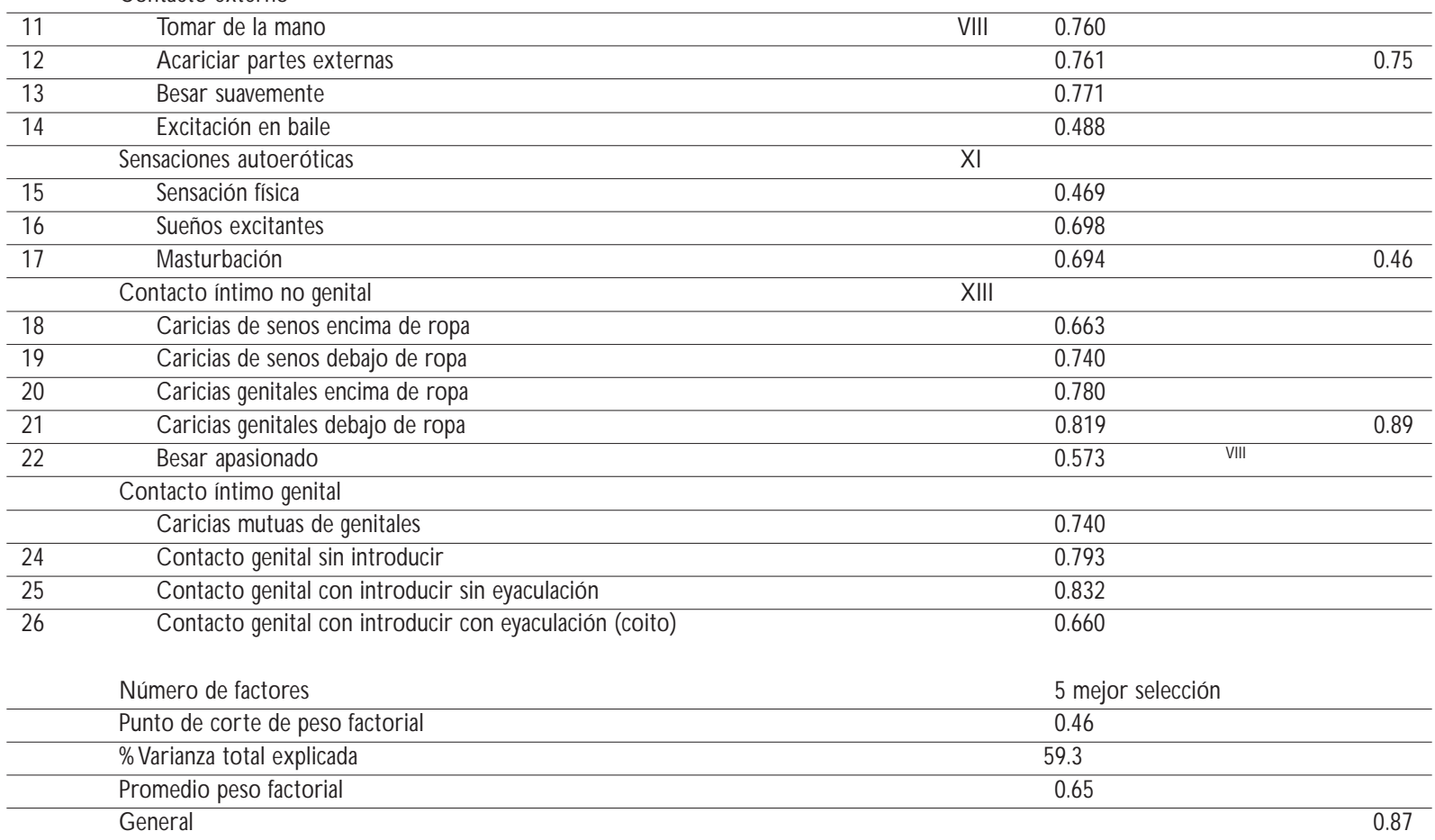


íntimo sino de Contacto externo; la Excitación en baile se integra a Sensaciones autoeróticas y el Noviazgo formal apareció como factor compartido, por una parte, con Noviazgo informal, y por otra, con Contacto íntimo.
En mujeres de preparatoria (cuadro III) se encontraron los factores II, V, VIII, XI y XIII, y sólo hubo similitud con mujeres de secundaria en el último de los factores. El comportamiento Llamar fue compartido con el factor II, semejando un factor I. Por

\section{FACTORES IDENTIFICADOS SEGÚN DOMINIOS, DIMENSIONES Y COMPORTAMIENTOS EN HOMBRES DE SECUNDARIA. Guadalajara, Jalisco, México}

\begin{tabular}{|c|c|c|c|c|c|}
\hline \multicolumn{2}{|c|}{$\begin{array}{l}\text { Dominios, Dimensiones } \\
\text { y Comportamientos }\end{array}$} & \multirow[t]{3}{*}{$\begin{array}{c}\text { Factor } \\
\text { valor }=>0.5\end{array}$} & \multirow[t]{3}{*}{$\begin{array}{l}\text { Peso } \\
\text { factorial }\end{array}$} & \multirow[t]{3}{*}{$\begin{array}{l}\text { Factor compartido } \\
\text { (valor: }>0.3<0.5 \text { ) }\end{array}$} & \multirow[t]{3}{*}{ Alpha } \\
\hline & SOCIAL & & & & \\
\hline Item & Fantasía & & & & \\
\hline 1 & Idea de estar solo & III & 0.671 & & \\
\hline \multirow[t]{2}{*}{2} & Escrito de lo que sientes & & 0.785 & & 0.69 \\
\hline & Imagen & & & & \\
\hline 3 & Te vea arreglada & & 0.805 & & \\
\hline 4 & Aparecer sexy & & 0.711 & & \\
\hline \multirow[t]{2}{*}{5} & Hacer cosas por & $\mathrm{VI}$ & 0.677 & III & \\
\hline & Búsqueda & & & & \\
\hline 6 & Conquistar & & 0.615 & III & \\
\hline \multirow[t]{2}{*}{7} & Llamar (teléfo no o recados) & & 0.754 & & \\
\hline & N oviazgo informal & & & & \\
\hline 8 & Salir solos & & 0.829 & & 0.84 \\
\hline \multirow[t]{2}{*}{9} & Juntarse para acariciarse & & 0.736 & & \\
\hline & N oviazgo formal & & & & \\
\hline \multirow[t]{3}{*}{10} & N oviazgo formal & & 0.738 & & \\
\hline & CORPORAL & & & & \\
\hline & Contacto externo & & & & \\
\hline 11 & Tomar de la mano & VIIII & 0,775 & XIII & \\
\hline 12 & Acariciar partes externas & & 0.746 & XIII & 0.89 \\
\hline 13 & Besar suavemente & & 0.731 & $X I I I$ & \\
\hline \multirow[t]{2}{*}{14} & Excitación en baile & & 0.706 & & \\
\hline & Sensaciones autoeróticas & $X I$ & & & \\
\hline 15 & Sensación física & & 0.646 & VIII & \\
\hline 16 & Sueños excitantes & & 0.810 & & \\
\hline \multirow[t]{2}{*}{17} & Masturbación & & 0.732 & XIII & 0.72 \\
\hline & Contacto íntimo no genital & XIII & & & \\
\hline 18 & Caricias de senos encima de ropa & & 0.802 & & \\
\hline 19 & Caricias de senos debajo de ropa & & 0.858 & & \\
\hline 20 & Caricias genitales encima de ropa & & 0.856 & & \\
\hline 21 & Caricias genitales debajo de ropa & & 0.897 & & 0.92 \\
\hline \multirow[t]{2}{*}{22} & Besar apasionado & & 0.826 & III & \\
\hline & Contacto íntimo genital & & & & \\
\hline 23 & Caricias mutuas de genitales & & 0.899 & & \\
\hline 24 & Contacto genital sin introducir & & 0.904 & & \\
\hline 25 & Contacto genital con introducir sin eyaculación & & 0.899 & & \\
\hline \multirow[t]{6}{*}{26} & Contacto genital con introducir con eyaculación (coito) & & 0.899 & & \\
\hline & $\mathrm{N}$ úmero de factores & \multicolumn{3}{|c|}{5 (valor propio $=1$ ) } & \\
\hline & Punto de corte de peso factorial & \multicolumn{3}{|c|}{0.50} & \\
\hline & \%Varianza total explicada & \multicolumn{3}{|c|}{70.62} & \\
\hline & Promedio peso factorial & \multicolumn{3}{|c|}{0.75} & \\
\hline & General & & & & 0.93 \\
\hline
\end{tabular}


otra parte, los comportamientos Hacer cosas por y Conquistar fueron compartidos con los factores relacionados con Noviazgos y con Sensaciones autoeróticas. El Besar apasionado tuvo factor compartido con el de Contacto externo (factor VIII).
En hombres de secundaria (cuadro IV) se encontraron los factores III, VI, VIIII, XI y XIII. Igualmente que en mujeres de preparatoria los comportamientos Hacer cosas por y Conquistar se comparten pero con el factor III, relacionado con Fantasía e Imagen. Igual

\section{Cuadro V \\ FACTORES IDENTIFICADOS SEGÚN DOMINIOS, DIMENSIONES Y COMPORTAMIENTOS EN HOMBRES DE PREPARATORIA. Guadalajara, Jalisco, México}

Dominios, Dimensiones

y Comportamientos

SOCIAL

Item Fantasía

\begin{tabular}{|c|c|c|c|c|}
\hline 1 & Idea de estar solo & III & 0.717 & \\
\hline 2 & Escrito de lo que sientes & & 0.711 & 0.71 \\
\hline \multicolumn{5}{|c|}{ Imagen } \\
\hline 3 & Te vea arreglada & & 0.716 & \\
\hline 4 & Aparecer sexy & & 0.677 & \\
\hline 5 & Hacer cosas por & VI & 0.717 & \\
\hline \multicolumn{5}{|c|}{ Búsqueda } \\
\hline 6 & Conquistar & & 0.773 & \\
\hline 7 & Llamar (teléfono o recados) & & 0.760 & \\
\hline \multicolumn{5}{|c|}{ N oviazgo informal } \\
\hline 8 & Salir solos & & 0.782 & 0.84 \\
\hline 9 & Juntarse para acariciarse & & 0.675 & \\
\hline \multicolumn{5}{|c|}{ N oviazgo formal } \\
\hline 10 & N oviazgo formal & & 0.696 & \\
\hline
\end{tabular}

CORPORAL

Contacto externo

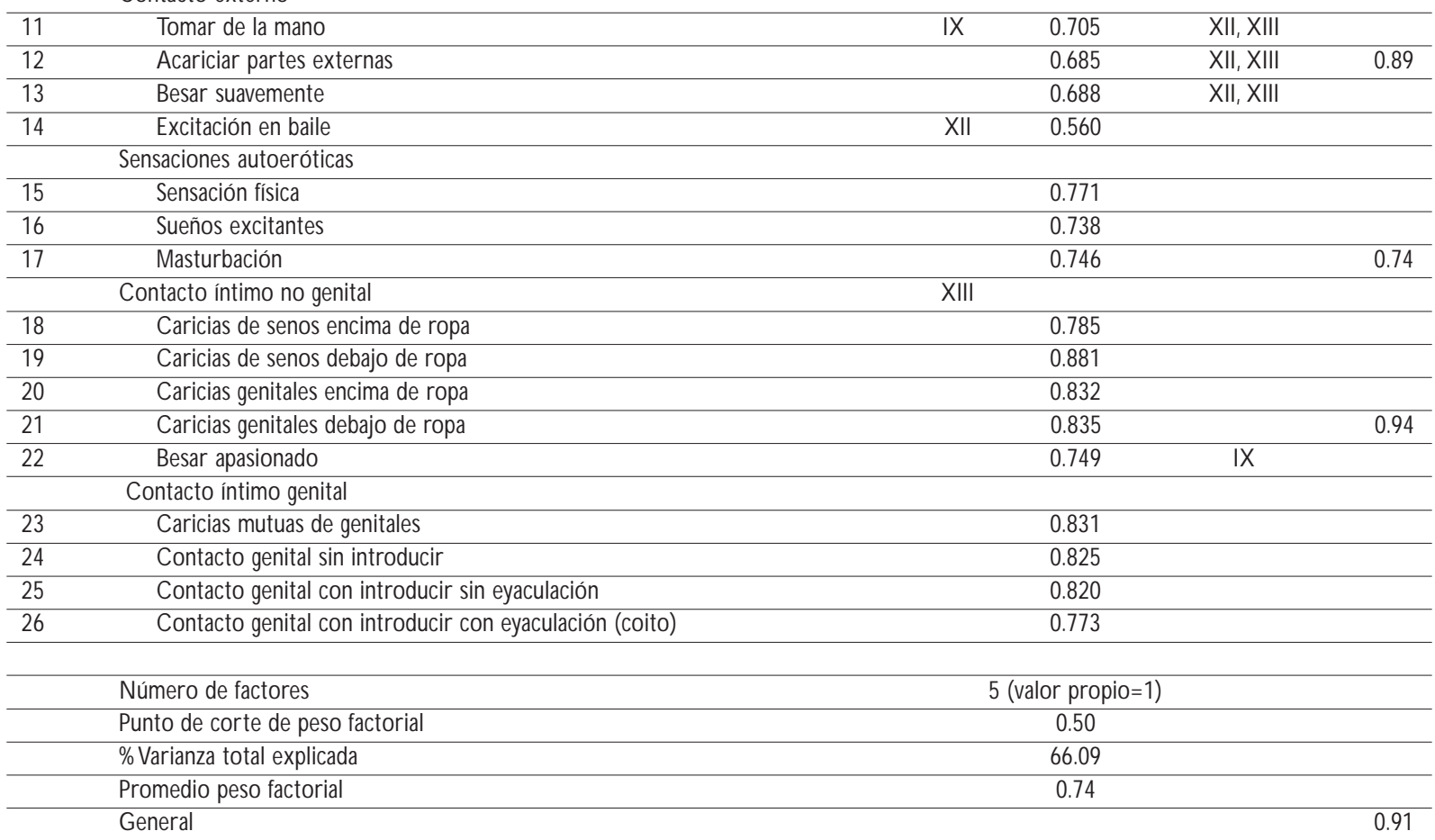


factor compartido incluye extrañamente Besar apasionado. Diversos comportamientos de Contacto externo y Masturbación tuvieron factores compartidos con Contacto íntimo (factor XIII)

En hombres de preparatoria (cuadro V) se encontraron los factores III, VI, IX, XII y XIII; esto se diferencia de hombres de secundaria sólo por la ubicación de un comportamiento, Excitación en baile. Los comportamientos de Contacto externo tuvieron factores compartidos relacionados con Sensaciones autoeróticas y de Contacto íntimo. El Besar apasionado se compartió con el factor Contacto externo (factor IX)

\section{Confiabilidad por consistencia interna}

En los cuadros II al V se observa que los coeficientes de Alfa de Cronbach, obtenidos para el total del instrumento en sus dos escalas para cada uno de los cuatro grupos, están entre 0.87 y 0.93 , que son resultados muy buenos. ${ }^{18} \mathrm{Al}$ examinar la consistencia interna de cada factor se encontraron cuatro con un valor menor de 0.60 (factor XI de Sensaciones autoeróticas en $\mathrm{Mp}$ ) y tres entre 0.60-0.70 (el factor III de Fantasía e Imagen en Hs, el factor $\mathrm{V}$ de Noviazgos en $\mathrm{Mp}$ y el factor $X$ de Sensaciones autoeróticas en Ms). En el resto, los valores son aceptables. La remoción de algún item no mejoró el valor de Alfa. El promedio de varianza de los items fue importante $(0.76,0.97,1.25 \mathrm{y}$ 1.20). En cuanto a las correlaciones de cada item con el total de items (corregidos) sólo 13 de los 104 tuvieron valores de correlación bajos, menores de 0.30 .

\section{Errores de medición y aplicación}

En cuanto a errores de medición en los items de validación, para analizar el subregistro, o sobrerregistro, se encontró que las mujeres tienen una frecuencia muy inferior de masturbación a la de los hombres, cualquiera que sea la calificación que hagan de ella y que sólo en mujeres de secundaria no hubo diferencias significativas $(\mathrm{x} 2=3.59 p=0.30)$ de la frecuencia de masturbación según la calificación de normalidad o no; en el resto sí fue significativa,-siendo más frecuente la masturbación cuando se calificaba como normal- o en el límite de lo normal.

En cuanto a errores de aplicación, al estudiar la confianza en la clave secreta en las sucesivas aplicaciones, se observó que casi alrededor de la mitad de los encuestados repetían claves y el resto no; y al comparar las características generales y comportamientos representativos entre los que repetían claves (supuestamente más veraces) y los que no repetían (supuesta- mente más desconfiados), no se encontraron diferencias significativas.

\section{Discusión}

Este cuestionario de estudio del comportamiento sexual de adolescentes surgió ante la inexistencia en México de un instrumento validado, no sólo sobre los comportamientos sexuales corporales, sino acerca de otros comportamientos sexuales sociales. Este enfoque se consideró que debía ser específico para cada género, por sus diversos desarrollos y se separó por edades expresadas en nivel escolar.

Hubo diversidad en lo que a edad y género se refiere, lo que permitió reflejar variaciones del fenómeno en estudio. El tamaño del grupo de entrevistados superó, en tres de los cuatro grupos, los 300 casos mínimos recomendados por Nunally, ${ }^{26}$ por lo que se puede generalizar a sus poblaciones equivalentes. Puesto que se construyó como Weinhard ${ }^{19}$ señala, a partir de la comunicación de sus experiencias, incorporando el lenguaje popular del adolescente urbano mexicano paralelo a los términos científicos, y como se usó una prueba piloto, se pudo lograr un instrumento con un alto grado de comprensión para esa población, manifestada en la baja consulta a los aplicadores y en las pocas respuestas sin contestar.

Los perfiles que los grupos presentan en el cuadro I y sus consecuentes cinco espacios factoriales visualizan semejanzas y diferencias que deben considerarse al analizar los resultados de la aplicación del instrumento a dichos grupos, generando en la práctica cuatro cuestionarios o a lo sumo tres, si se considera la gran similitud de factorización en los hombres. Esto muestra la necesidad de comprender diferencialmente, por nivel escolar y por género, las respuestas de adolescentes e inferir el significado que puedan tener para el desarrollo de una educación sexual.

Si se analiza la separación en los dos dominios originales, social y corporal, en ambos grupos de hombres y en las mujeres de preparatoria, pero no así en mujeres de secundaria, estos podrían corresponder a los cambios en componentes individuales-fisiológicos y sociales a través de la adolescencia, expresados por Fisher ${ }^{4}$ siguiendo la teoría de la secuencia del comportamiento sexual, y en donde se confunden lo social y corporal inicialmente en mujeres y seguramente tambien en hombres de edades menores a las estudiadas

En el Dominio social existe similitud de factores en los hombres, lo que lleva a preguntarse si corresponde a una construcción social general para este género. ${ }^{30}$ Aparece a) un factor III que muestra una 
disociación entre las dimensiones de Fantasía-Imagen de la de Búsqueda, lo que quizá parece identificar un esquema de comportamiento más eficiente, ya que la Búsqueda (¿sistemática?) está ligada o aparece concatenada a los noviazgos (¿novio/a en espera?), y b) un factor VI. ¿Integración deliberada de noviazgos con fines de acercamiento más íntimo? Por el contrario, en las mujeres los factores son diversos, lo que puede estar significando asignaciones sociales diferenciales en la edad y situación escolar o, por el contrario, quizás explicarse por una procedencia diferente de ambas poblaciones.

Aparece el factor I en mujeres de secundaria conformado en una unidad de pensar-actuar; parece ser sólo un juego en este proceso de aprendizaje de acercamiento a la persona deseada y que persiste en las mujeres de preparatoria (factor II) excepto en el comportamiento "LLamar", que se integra a los Noviazgos. Westhey ${ }^{31}$ también encuentra en las adolescentes tempranas un mayor y diferencial uso del teléfono que los hombres. Parece ser que las adolescentes tempranas tienen comportamientos más cercanos al modelo social de papeles sexuales de una sociedad tradicional, donde se separan comportamientos de Fantasía, Imagen y Búsqueda del Noviazgo informal o formal, comparados con hombres de secundaria en quienes no ocurre. Esto es diferente a los hallazgos de Nelson, ${ }^{32}$ quien encontró más tradicionalismo en actitudes hacia la conducta sexual en hombres adolescentes tempranos que en mujeres.

Las mujeres de secundaria estructuran un factor IV acerca de la dimensión Noviazgo informal y que puede corresponder a lo que Roche ${ }^{10}$ caracteriza con dos comportamientos relacionados: salir con una pareja sin afecto o con afecto pero sin amor, y tener comportamientos livianos o pesados corporales sin llegar al coito, y un factor VII en donde el Noviazgo formal integra contactos físicos externos. Los Noviazgos informal y formal estarían separados en las mujeres de secundaria en razón de ese comienzo más tardío de actividad sexual (no puberal) femenino, que Useche también encuentra en adolescentes colombianos. ${ }^{33} \mathrm{En}$ las mujeres de preparatoria, por el contrario, un factor $\mathrm{V}$ muestra una integración (¿o confusión?) de ambos Noviazgos.

Esta distinción por género al inicio de la adolescencia, es encontrada en la revisión de $\mathrm{McCabe}^{34}$ de autores como Maddock y McCandeless, quienes destacan el dating (o salir con) como una aproximación psicobiológica en busca de relaciones físicas en el adolescente y psicoafectivas en busca de relaciones de amor en la adolescente. Las implicaciones pueden significar un embarazo adolescente producto de tan diversas expectativas. Algo semejante encuentra Roche ${ }^{10}$ en las primeras etapas del dating: diferencias de género a favor en los hombres de una mayor presencia de Contactos íntimos.

Si se analiza una dimensión como la Búsqueda en los diferentes grupos se encuentra que en la adolescente menor sólo es parte de un juego de Fantasía e Imagen, en las mayores el camino al Noviazgo informal y formal; para los hombres es camino para un Noviazgo indistinguible.

Entre los factores encontrados en el Dominio corporal, la dimensión Contacto externo corresponde a lo que diversos autores señalan como fases de contacto físico inicial del encuentro de una pareja ${ }^{7,8,9,10} \mathrm{y}$ que sólo en mujeres de secundaria se manifiesta, integrándolas a la dimensión Noviazgo formal, que parece ser el que autoriza esos contactos físicos iniciales (factor VII); un factor VIII en mujeres de preparatoria y hombres de secundaria, que agrupa comportamientos independientes de dimensiones como los Noviazgos y las Sensaciones autoeróticas, y un factor IX semejante en hombres de preparatoria, pero sin el comportamiento Excitación en baile.

Los factores relacionados con la dimensión Sensaciones autoeróticas (factores X,XI,XII) constituyen en muchos adolescentes las primeras experiencias exploratorias o psicosensoriales sexuales..$^{9}$ En mujeres, integra comportamientos interpersonales como la Excitación en bailes; ${ }^{35} \mathrm{y}$ en hombres de preparatoria, quizás como una alternativa de experiencias, de un legítimo acercamiento corporal en lo externo o en lo íntimo.

Destaca el factor XIII, referido a las prácticas sexuales señaladas por autores como comportamientos sexuales precoitales pesados ${ }^{10}$ y coitales, que resulta ser el único factor que es atributo de ambos géneros y grupos escolares, lo cual habla de una homogeneidad de lo más íntimo; del mismo modo como podemos ver en otros factores la heterogeneidad en lo más público. La teoría del script o guión sexual de Gannon y Simon, ${ }^{36}$ que señala que "los scripts están envueltos en el significado de estados internos, organizando la secuencia de actos sexuales específicos, decodificando situaciones nuevas, enmarcando los límites de las respuestas sexuales y ligando significados de aspectos vitales no sexuales a experiencias sexuales específicas", ayuda a comprender para la sociedad de los adolescentes en estudio que los contactos íntimos estén tan homogéneamente pautados. Pero al contrario de otros hallazgos en otros países, no aparecen estos comportamientos ligados estrechamente al Noviazgo formal o informal. ${ }^{8,10}$ También estos hallazgos difieren de los de Roche ${ }^{10}$ en que los comporta- 
mientos de mayor intimidad se asocian a estados de "salir con" (dating) la pareja única enamorada, que en nuestra investigación corresponde a Noviazgo formal, y que se unen en hombres y en mujeres de preparatoria sólo al Contacto externo. Quizá en una sociedad con rasgos tradicionales, como en la que se encuentra la población en estudio, tales relaciones íntimas aún son estigmatizadas.

La validación del cuestionario implicó usar también criterios de confiabilidad. Los altos valores alcanzados tanto en hombres como mujeres, de secundaria o preparatoria, hablan de que los items forman parte de una escala donde hay alta correlación entre éstos y aun identifican los dominios social y corporal con valores aceptables, producto de la alta variabilidad y correlación de los items. Los valores superiores a 0.90 , a criterio de DeVellis, ${ }^{20}$ en lo corporal, estarían indicando redundancia de items que podrían ser reducidos, ya que en la dimensión Contacto íntimo están influyendo seguramente comportamientos muy similares (pero que se establecieron para identificar cuáles predecían, en su uso longitudinal, el coito ). El factor con valor no aceptable (factor XI) es por la muy baja correlación entre los items 15 y 17 ( 0.12) y entre el 15 y $16(0.23)$ lo que obligaría a agregar otros items, ya que en general la dimensión Sensaciones autoeróticas tienen valores poco deseables, y son los valores más bajos en mujeres; y se podría considerar que aparecieron respuestas más controladas en comportamientos estigmatizados como es la masturbación femenina.

Las limitaciones derivan de ser un estudio de comportamientos, muchos de carácter privado, por lo que pudo existir la desconfianza en la clave secreta usada para el estudio longitudinal y por ello se estudió en las aplicaciones siguientes el efecto del anonimato que se pretendía con la clave secreta, pero no se encontraron diferencias significativas en los comportamientos sexuales entre ellos, por lo cual esta limitación parece no ser tal; hasta ser un subregistro de comportamientos estigmatizados para cierto género, como masturbación en la mujer, que, dados los hallazgos, pudo ser cierta, o al representar alguna dimensión de autoestima (especialmente para hombres) pudo haber sobrerregistro (se observó que en secundaria [adolescencia inicial], aun cuando las mujeres tienen una pubertad más temprana, hubo mayores porcentajes de comportamientos sexuales en los hombres debido a un rol social asignado o quizá al sobrerregistro mismo).

Este análisis de tipo exploratorio produjo una estructura adecuada a su objetivo, es decir, de obtención de pocos factores en cada grupo, altamente definidos para cada género y grupo escolar y que explicaron un porcentaje importante de la varianza total. Al no pretenderse un análisis factorial confirmatorio, los dominios y dimensiones iniciales sólo fueron para generar un rango de items que abarcara el contenido en estudio; los resultados, en todo caso, con excepción de los de mujeres de secundaria comprobaron los dominios pero no las dimensiones, excepto Contacto íntimo. Los resultados encontrados en el análisis de constructo apuntan a que el cuestionario detecta constructos latentes distintos entre géneros y entre edades (a las que corresponden los niveles escolares) y también dentro del género femenino, pero no en el masculino. Los pesos factoriales alcanzados implicaron correlaciones altas entre los items y sus constructos latentes, y los factores alcanzaron valores de confiabilidad aceptables por lo que, en conclusión, se puede decir que el proceso de validación, por validez de constructo y de consistencia interna, obtuvo resultados que legitiman el instrumento para poblaciones equivalentes en edad, género y condición socioeconómica, pero respetando la factorización específica de cada grupo y las limitaciones señaladas. Por lo tanto, la aplicación del cuestionario debe implicar un análisis diferencial de género así como entre las mujeres, y se debe considerar esta diversidad en los procesos de educación sexual.

\section{Agradecimientos}

Esta investigación fue parcialmente financiada por la Fundación Mexicana para la Salud y por la Fundación Carnegie (Proyecto FP 0056), y apoyada por la Unidad de Investigación Epidemiológica y en Servicios de Salud del Instituto Mexicano del Seguro Social, y por el Centro Universitario de Ciencias de la Salud de la Universidad de Guadalajara. Los autores agradecen la participación, en el trabajo de campo, de las Dras. Martha Villaseñor y Amparo Tapia, de la trabajadora social Bertha Nuño, del psicólogo Javier Becerra y del Dr. Alberto Villaseñor.

\section{Referencias}

1. Friedman HL. C hanging patterns on adolescent sexual behavior: C onsequences for health and development. J A dolesc $H$ ealth 1992;5:345-350.

2. Maddaleno M, Silber T. Un punto de vista epidemiológico de la salud del adolescente en América Latina. J Adolesc Health 1993;14:655-633.

3. Byrne D, Kelley K.Alternative approaches to the study of sexual behavior. Londres: Law rence Erlbaum Associates Publishers, 1986:1-12.

4. Fisher W A. Psychological approach to human sexuality:The sexual behavior sequence. En: Byrne D, Kelley K. Alternative approaches to the study of sexual behavior. Londres: Lawrence Erlbaum Associates, 1986: 131-171. 
5. Sorensen RC. Adolescent sexuality in contemporary America. Tarrytown N ueva York:W orld, 1973.

6. Schawartz I. Sexual activity prior to coital initiation: A comparison between males and females. Arch Sex Behav 1999; 128(1):63-69.

7. Collins JK. A dolescent dating intimacy: N orms and peer expectatives. J Youth Adolescence 1974;3:317-328.

8. Rodgers JL, Billy JO G U U dry JR. The rescission of behaviors: Inconsistent responses in adolescent sexuality data. Soc Sci Res 1989; 11:280.

9. Grace E, Strasburger V. N ormal sexuality. En: McA narney E.Textbook of adolescent medicine. México, D.F.:W.B. Saunders Company, 1992:650-653.

10. Roche J. Premarital sex:Attitudes and behavior by dating stage. Adolescence 1986;31(81) 107-121.

11. Silva M. Relaciones sexuales en la adolescencia. Santiago, Ediciones Universidad Católica de Chile, 1991:25-26.

12. Montgomery M, Sorell G. Love and dating experience in early and middle adolescence: $G$ rade and gender comparisons. J A dolescence 1998; 21:677-689.

13. Consejo $\mathrm{N}$ acional de Población. Encuesta $\mathrm{N}$ acional sobre Sexualidad y Familia en Jóvenes de Educación Media Superior México. México, D.F: Conapo, 1988.

14. Morris L, Bailey $P, N$ úñez L, Monroy A, W hatney D, C árdenas C.Young Adult Reproductive Health Survey in Two Delegations of Mexico City. México D.F: CORA FHIAMIDEM CDC, 1987:1-410.

15. G arcía J, Figueroa JG Práctica anticonceptiva en adolescentes y jóvenes del área metropolitana de la ciudad de México. Salud Publica Mex 1992; 34 (4): 413-426.

16. CO N ASIDA. Comportamiento sexual en ciudad de México. Encuesta 1992-1993. México, D.F.: 1995

17. Fleiz C, Villatoro J, Medina E,Alcántara E, N avarro C, Blanco J. Conducta sexual en estudiantes de la ciudad de México. Salud Mental 1999;22: 14-19.

18. Dare $0, C$ leland J. Reliability and validity of survey data on sexual behavior. IUSSP Seminar on "Aids prevention in developing countries:The contribution of demography and social science". París:Alan Guttmacher Institute, 1993.

19.W einhardt L, Forsyth A, C arey M, Jaworsky B, D urant L. Reliability and validity of self-report measures of HIV- related sexual behavior: Progress since 1990 and recommendations for research and practice. Arch Sex Behav 1998;27(2): 155-180

20. DeVellis R. Scale development:Theory and applications. N ewbury Park, CA: Sage, 1991.

21. Katchadourian H. La sexualidad humana. Un estudio comparativo de su evolución. México, D.F.: Fondo de Cultura Económica, 1983.

22. Polit D. Investigación científica en ciencias de la salud. México DF: Ed. Interamericana,1995:313-315.

23. Alvarez R. Estadística multivariante y no paramétrica con SPSS. A plicación a las ciencias de la salud. Madrid: Díaz de Santos, 1995:243-244.

24. Kim JO, Mueller CHW. Factor Analysis. Statistical methods and practical issues. Q uantitative applications in the Social Sciences Series. N ewbury Park: Sage, 1978;14: 74-77.

25. Gable R. Instrument development in the affective domain. Boston: Kluwer-N ijhoff, 1986.

26. N unally J.Teoría Psicométrica. México D F:McG raw Hill,1995;254,600603.

27. C armine E, Zéller R. Reliability and validity assesement. Q uantitative applications in the social science series. N ewbury Park: Sage, 1979;17: 44-47.

28. N orusis M. SPSS Professional Statistics 6.1. USA: SPSS, 1993: 52-53.

29. Masters W, Johson V, Kolodny R. La Sexualidad Humana. Barcelona, España: Grijalbo, 1987;36.

30.W eeks J. Sexualidad. México DF.: Ed. Paidós,1998:28-32.

31. Westhey 0 , Jenhiw D, Robbs J, W illiams I. Sexual development and behavior in black preadolescent adolescence 1984; 19(75): 557-569.

32. N elson C, Jeith J. Comparison of female and male early adolescent sex role attitude and behavior development.Adolescence 1990; 25(97): 184-203.

33. Useche B, Villegas M. Sexual behavior of colombian high school students. Adolescence 1990;25(98): 291-304

34. McC abe M.Toward a theory of adolescent dating.A dolescence 1984;19 (73):159-170,

35. Brown R, Crome B.Adolescent sexuality. En: San Filippo, ed. Pediatric and adolescent gynecology. Filadelfia (PA):W B Saunders, 1994. 36. Gannon J, Simon W. Sexual conduct. Chicago:Aldine, 1973. 


\section{ANEXO 1}

El cuestionario final comprendió preguntas dirigidas a las características sociodemográficas de los encuestados (edad, género, nivel escolar, trabajo, con quién vivía, escolaridad materna y trabajo de padres).A cerca del comportamiento sexual se preguntaba si no se había producido o si se había producido el mes anterior 0 entre dos a seis meses antes, 0 hacía más de seis meses (en el caso del cuestionario inicial). Se integraron en el Dominio social 10 items distribuidos en las dimensiones: Fantasía (items 1 y 2), Imagen (items 3 al 5) Búsqueda (items 6 y 7), N oviazgo informal (items 8 y 9 ) y $\mathrm{N}$ oviazgo formal (item 10). El Dominio corporal se integró por 16 items distribuidos en las dimensiones: Contacto externo (items 11 al 14), Sensaciones autoeróticas (items 15 al 17) Contacto íntimo no genital (items 18 al 22) y Contacto íntimo genital (items 23 al 26):

1. ¿Te ha pasado por la mente la idea de estar sólo con ella [él] en la playa u otro lugar?

2. ¿Has escrito lo que sientes por ella [él] en un diario personal, o le has hecho poemas, canciones o dibujos?

3. ¿Te has preocupado porque te vea arreglado [a]?

4. ¿Has tratado de quedar bien con ella(él) apareciendo muy "chingón" o muy galán[muy sexy o muy coqueta]?

5. ¿Has intentado hacer por ella [él] cosas que no harías por nadie?

6. ¿Te has acercado para conquistarla [0]?

7. ¿Le has llamado por teléfono o le has mandado recaditos?

8. ¿H an salido solos al cine 0 a una fiesta?

9. ¿Te has juntado con una [un] "chava" [0] sólo para pasar el rato, abrazarse, besarse o acariciarse?

10. ¿Tienes actualmente novia [0] formal 0 en serio?

11. ¿Te has tomado de la mano con ella [él]?

12. ¿Le has acariciado la mano, la cara o cuello?

13. ¿Le has besado suavemente?

14. ¿Te has excitado cuando has bailado con ella [él]?

15. ¿Has sentido que se te acelera el corazón o que te sube la sangre o que no te salen las palabras al verla[0]?

16. ¿Has tenido "sueños húmedos o vaciarte" [o excitantes]?

17. ¿H as tenido la experiencia de masturbación o "jalártela" "jugar o acariciar tus partes íntimas"]?

18. ¿Has [Te han] acariciado por encima de la ropa sus(tus) senos?

19. ¿Has [Te han]) acariciado por debajo de la ropa sus [tus] senos?

20. ¿Has [Te han] acariciado por encima de la ropa sus[tus] partes íntimas?

21. ¿Has[Te han] acariciado por debajo de la ropa sus[tus] partes íntimas?

22. ¿La[lo] has besado apasionadamente 0 dado un "beso húmedo"?

23. ¿Se han acariciado mutuamente por debajo de la ropa las partes íntimas?

24. ¿Has tenido contacto íntimo sexual, pero sin que haya introducción o"sin meter"?

25. ¿Has tenido contacto íntimo con introducción o"con meter", pero sin eyaculación o"sin derramarse o vaciarse dentro"?

26. ¿H as tenido coito, o sea contacto íntimo con introducción o "meter", y con eyaculación o "derramarse o vaciarse dentro"?

Las corchetes [ ] indican con qué frase se cambió el texto de hombres en el cuestionario de mujeres y las comillas (" ") incluyen el término popular del comportamiento. Se inició con el número del item. 